\title{
Comparative Evaluation of Postoperative Pain and Periapical Healing After Root Canal Treatment Using Three Different Base Endodontic Sealers - A Randomized Control Clinical Trial
}

\section{Akshay Khandelwal ( $\sim$ akshay92khandelwal@gmail.com )}

Department of Conservative Dentistry and Endodontics, Saveetha Dental College, Saveetha Institute of Medical and Technical Sciences, Saveetha University, Chennai, Tamil Nadu Jerry Jose

Department of Conservative Dentistry and Endodontics, Saveetha Dental College, Saveetha Institute of Medical and Technical Sciences, Saveetha University, Chennai, Tamil Nadu

Ajitha Palanivelu

Department of Conservative Dentistry and Endodontics, Saveetha Dental College, Saveetha Institute of Medical and Technical Sciences, Saveetha University, Chennai, Tamil Nadu

\section{Kavalipurapu Venkata Teja}

Department of Conservative Dentistry and Endodontics, Saveetha Dental College, Saveetha Institute of Medical and Technical Sciences, Saveetha University, Chennai, Tamil Nadu

\section{Research Article}

Keywords: root canal obturation, root canal sealants, periapical periodontitis, periapical healing, postoperative pain

Posted Date: June 30th, 2021

DOI: https://doi.org/10.21203/rs.3.rs-622404/v1

License: (c) (i) This work is licensed under a Creative Commons Attribution 4.0 International License. Read Full License 


\section{Abstract}

Background》 Endodontic Sealers come in direct contact with periapical tissue through the apical foramen and lateral canals, influencing the postoperative pain and periapical healing. The aim of the present study was to evaluate and compare postoperative pain and periapical healing after root canal treatment using different base endodontic sealers.

Methods $\varangle$ Primary root canal treatment was initiated in 63 patients diagnosed with necrotic pulp and apical periodontitis, followed by which the endodontic sealers used for obturation were selected based on the random allocation of the participants to the following groups; Tubli-Seal, AH Plus and BioRoot RCS. Postoperative pain was recorded by using $100 \mathrm{~mm}$ visual analog scale at $24 \mathrm{~h}, 48 \mathrm{~h}, 72 \mathrm{~h}$ and $7 \mathrm{~d}$ after obturation. Digital periapical radiographic evaluation was done to assess rate of periapical healing at baseline, 1, 3 and 6 months. Statistical analysis was done using Kruskal Wallis test and one-way ANOVA and p-value of less than 0.05 was considered as significance level.

Results $\varangle$ Significant reduction was seen in the size of periapical lesions in all the study groups at 3 and 6 months ( $p \otimes 0.05)$. The mean difference in the size of periapical lesions for BioRoot RCS was 4.05, 10.22, for AH Plus was 2 3.86, 9.80 and Tubli-Seal were 6.27, 13.41 at 3 months and 6 months respectively. The mean pain scores at $24 \mathrm{~h}$ for Tubli-Seal, AH Plus, BioRoot RCS were 17.94 $\pm 11.35,11.57 \pm 11.18$ and $4.73 \pm 7.72$. At $48 \mathrm{~h}$ were $5.26 \pm 9.04,1.57 \pm 3.74$ and $1.57 \pm 3.74$ respectively. The mean pain score at 72 $\mathrm{h}$ for Tubli-Seal was $2.63 \pm 7.33$ whereas none of the patients had reported pain in AH Plus and BioRoot RCS group. None of the patients had pain $7 \mathrm{~d}$ after treatment.

Conclusions $₫$ BioRoot RCS showed less postoperative pain compared to AH Plus and Tubli-Seal. BioRoot RCS showed better periapical healing compared to AH Plus and Tubliseal at 3- and 6-months interval respectively.

Trial Registration: Registration of this trial was done prospectively in Clinical trials registry - India (CTRI) with registration number (CTRI/2018/10/015919) dated 08/10/2018.

\section{Background}

Apical periodontitis is an inflammatory lesion around the periapical region and can have significant influence on the endodontic treatment prognosis. Teeth with apical periodontitis are considered to be an entombment of various pathological flora which can influence the success rate of the treatment (1). The aim of endodontic treatment in such scenarios is to maintain an adequate biological environment allowing physiological healing to occur (2). This is achieved by the complete disinfection of root canal system using root canal irrigants such as sodium hypochlorite $(\mathrm{NaOCl})$ and ethylenediaminetetraacetic acid (EDTA) which actively act on the organic and inorganic portion of the smear layer matrix seen to be a reservoir for various microorganisms (3). Regarding these cases the primary success of conventional endodontic treatment depends on the elimination of organisms responsible for causing periapical pathology. Root canal obturation plays a crucial role in endodontic therapy since it reduces bacterial 
contamination by preventing coronal leakage and by sealing the apex from periapical tissue fluids and it also entombs the remaining microbes in the canal preventing further disease (4).

Periapical healing is seen to be the structural and functional replacement of the bone considered to be an intricate interplay between the osteoclasts and osteoblasts allowing the bone formation to occur seen to be influenced by the host's intrinsic and extrinsic mechanisms (5). Root canal sealer is a local factor which interferes with the healing of periapical tissues by leaching through the apical foramen and lateral canals (6). Based on the composition of root canal sealers such as zinc oxide-eugenol formulations, calcium hydroxide sealers, glass ionomer sealers, resin-based (epoxy resin or methacrylate resin) sealers and recently introduced bioceramic based sealers the periapical healing can be influenced by changing the rate of bone deposition as well as creating an enhanced environment for remineralization to occur (7).

Postoperative pain is considered to be another significant clinical outcome exhibiting a multifactorial response to treatment related factors such as maintaining the working length to the apical constriction, finishing the endodontic treatment in single visit or multiple visit, instrumentation technique and the type of endodontic sealer used for obturation (8). Postoperative pain usually ranges from $3 \%$ to $58 \%$ based on the individual's pain perseverance and stimulus (9). Such pain occurrence is mainly due to mechanical, chemical or microbial injury to the periapical tissues (10). Root canal sealers can play a crucial role in this regard by coming in contact with the periapical tissues through apical foramen and lateral canals causing a localized inflammation with a direct influence on the degree of inflammation based on the composition of the sealer in turn influencing postoperative pain levels (11).

The present study aimed to address both these issues by conducting a comparative evaluation of the incidence and intensity of postoperative pain and post obturation healing of periapical lesions after primary root canal treatment using representatives of different base endodontic sealers such as zinc oxide eugenol sealer (Tubli-Seal), resin-based sealer (AH Plus) and bioceramic sealer (BioRoot RCS) in patients with apical periodontitis. The null hypothesis was considered that there was no significant difference in the incidence and intensity of postoperative pain and periapical healing after root canal treatment using BioRoot RCS, AH Plus and Tubli-Seal as endodontic sealers.

\section{Methods}

The present study adhered to the Consolidated Standards of Reporting Trials (CONSORT) statement of reporting (Additional file 1). Ethical approval was obtained from the Institutional Review Board (SRB/SDMDS11/170DS/09). The protocol for the present clinical trial was registered with the clinical trials registry - India (CTRI) with registration number (CTRI/2018/10/015919) before the clinical trial began. All the patients consented to an informed consent form prior to the start of the treatment which mentioned the details of the present study as well as benefits and risks of the study.

\section{Trial Design}


The present study was conducted under a university setting which followed a double-blinded parallel randomized clinical trial design with allocation ratio of 1:1 in which the patient and the assessors were blinded from the process. The treating operator could not be blinded since treatment protocol with different endodontic sealers could not be concealed from the operator used in the present study.

\section{Sample size calculation}

A priori sample size calculation was done using $G *$ Power 3.1.2 software based on a previously published study (12). Using a one-way ANOVA fixed effects omnibus model $(a=0.05,1-\beta=0.95, f=0.05)$, the minimum sample size calculated was 18 per group. Expecting attrition of the sample during follow-up, the sample size was adjusted by $10 \%$ to 63,21 per group.

\section{Study groups}

For the present study, 3 groups were taken which were representatives of different base endodontic sealers; Group 1: ZOE based sealer (Tubli-Seal, Sybron Endo, Romulus, MI), Group 2: epoxy resin-based sealer (AH Plus, Dentsply DeTrey, Konstanz, Germany), Group 3: Bioceramic based sealer (BioRoot RCS, Septodont, USA)

\section{Inclusion/Exclusion criteria}

Participants undergoing endodontic therapy in maxillary anterior teeth within the age group of 18-60 years categorized under American Society of Anesthesiologists (ASA - 1) giving a tooth diagnosis of necrotic pulp with chronic apical periodontitis confirmed using sensibility test [cold test (1, 1, 2tetrafluoroethane. Hygienic Endo-Ice Green [Endo-Ice]; Coltene Whaledent, Cuyahoga Falls, $\mathrm{OH}$ ) and electrical pulp testing (Analytic Technology Pulp Tester, Analytic Technology, Redmond, Wash)] with a periapical index (PAI) score of 2 or more diagnosed using digital periapical radiograph as well as a patient's VAS Score of $30 \mathrm{~mm}$ and above were taken into inclusion criteria for the present study since the present study aimed to evaluate two clinical parameters; post endodontic pain and periapical healing. Patients classified other than ASA - 1, immature permanent tooth, tooth exhibiting endodontic-periodontic lesions, dystrophic calcifications within the tooth as well as more than $20^{\circ}$ curvature, pregnant or lactating women, root fracture cases, patients who consumed analgesics $12-24 \mathrm{~h}$ before the primary root canal treatment were excluded from the present study.

\section{Randomization}

Computer-generated random table of numbers was conducted using an online service (random.org) was used for the randomization process to assign the participants to different study groups. Block randomization method was advocated using the SNOSE (sequentially numbered, opaque, sealed envelopes) method for allocation concealment. 2 experienced endodontists (V.T, J.J) who were not involved in the treatment process assessed these periapical radiographs and came to a consensus regarding the scoring outcome for the size of the lesion. In case of disagreement, a third specialist who had sufficient experience for more than 10 years in interpreting radiographs was consulted to achieve an 
agreement. A paper containing the randomized group number was sealed in the dark-colored envelope containing the respective serial and treatment protocol for only the sealer groups prepared by the respective third person (A.P). Study numbers were sequentially assigned to patients by an individual not related to the present study. The envelope was opened once the intervention was assigned. Respective treatment was carried out based on the group assigned.

\section{Treatment protocol}

The treatment procedure was conducted by a single operator (A.K). All the groups underwent the same protocol. Prior to the treatment, a digital radiographic evaluation was conducted using a customized grid with paralleling technique. Lesion sizes which gave a PAI score 2 or more were recruited for the present study. The treatment protocol was explained to all the participants and informed consent was obtained. A total of 63 patients were recruited in this study, who fulfilled the above-mentioned selection criteria. The teeth were isolated using a rubber dam using a single tooth isolation technique, caries excavation was conducted and a pre-endodontic build-up was done using composite resin (3M Filtek, 3M ESPE, USA), if required. Access cavity preparation was conducted using Endo Access Kit (Dentsply Maillefer, Ballaigues, Switzerland) followed by which debridement of pulp chamber contents was done using a spoon excavator and $3 \%$ sodium hypochlorite ( $\mathrm{NaOCl}$ ) (Prime dental products, Thane, India). An ISO size $10 \mathrm{~K}-$ File (Mani Corp. Japan) was used to obtain for initial patency filing and working length was recorded using an electronic apex locator (Root ZX II, J. Morita, MFG. Corp. Kyoto, Japan) such that it was measured at $0.5 \mathrm{~mm}$ short of the apical canal terminus (' 0 ' reading). The confirmation of the working length was done by using digital periapical radiograph.

The canals were shaped using Protaper Gold (PTG, Dentsply Maillefer, Ballaigues, Switzerland) for all the teeth. The apical preparation was carried out by using ISO stainless steel hand K files (Dentsply Sirona, Ballaigues, Switzerland) starting with the file which was initially binding to the canal till the working length, the final instrumentation was carried out 3 sizes larger than the initial binding file followed by which a similar taper instrumentation technique using Protaper Gold till the working length. During the instrumentation process, $3 \%$ sodium hypochlorite ( $\mathrm{NaOCl}$, Prime Dental, India) was used during each cycle of instrumentation and the canal patency was maintained by passing ISO 15 No. K file approximately $1 \mathrm{~mm}$ beyond the determined working length after each instrumentation cycle. To effectively remove the smear layer, irrigation of $17 \%$ ethylenediaminetetraacetic acid (EDTA, Anabond Stedman, Kanchipuram, India) followed by a final irrigation of $3 \% \mathrm{NaOCl}$ was conducted. All the irrigation process was conducted using a 30-gauge double side vented needle (Neoendo, Orikam Healthcare, India) and $2 \mathrm{ml}$ syringe barrel (Dispovan, India). During the irrigation process each cycle was intermittently activated using sonic activation for a period of $60 \mathrm{~s}$ (EndoActivator, Dentsply Sirona, USA).

Post biomechanical preparation procedure, the canals were dried using sterile paper points according to corresponding taper and freshly mixed calcium hydroxide paste was placed into the prepared canals using a lentulospiral (Dentsply Maillefer, Ballaigues, Switzerland) followed by which a temporary seal was done using intermediate restorative cement (IRM, Dentsply Sirona, USA). The patients were recalled 
after a week and patients who were asymptomatic exhibiting a VAS Score of 0 and dry canals on evaluation with sterile paper points after removal of the intracanal medicament were further treated. The teeth were obturated according to the randomly allocated groups, Group 1: Tubli-Seal; Group 2: AH Plus; Group 3: BioRoot RCS.

Manufacturer's instructions were followed for mixing the respective sealer on a sterile glass slab. The apical extent of the master cone was confirmed radiographically by digital periapical radiograph. The canals were dried using sterile paper points and were coated with the sealer using lentulospiral (Dentsply Maillefer, Ballaigues, Switzerland) in a slow speed handpiece (NSK Corp., Tochigi, Japan) followed by which the obturation process was performed with respective sealers using the lateral compaction technique.

Post-treatment occlusal reduction of $1 \mathrm{~mm}$ was done in all the treated teeth and permanent restorations was done with composite resin (Filtek Z 350, 3M ESPE, USA) and the periapical healing and postoperative pain was assessed. All the clinical procedures were performed by one operator of similar endodontic clinical experience. A partial coverage or a full coverage prosthetic management was done for all the teeth as indicated.

\section{Outcome assessment}

\section{Periapical healing assessment}

All the digital periapical radiographs were carried out using parallel cone technique using RVG sensor (Carestream Dental LLC, Atlanta, GA) with the help of a sensor positioning system (Bluedent, India) and was evaluated for baseline data. The data was analyzed by two experienced endodontists (V.T, J.J) who were not involved in the treatment protocol such that prior images were seen so the interobserver agreement was seen at 0.90 using Cohen kappa $(p<0.05)$. The presence or absence of sealer extrusion was also noted. The size of the periapical lesion was calculated with the help of a grid, $\mathrm{X}$-ray mesh gauge (Bluedent, India) such that the entire proximity of the periapical lesion was covered under the mesh gauge and the same observers (V.K, J.J) confirmed the size of the lesion at repeated intervals and mean scores were taken. Subsequent radiographs were taken for each patient at 1, 3 and 6 months using digital periapical radiographs and evaluated using the above mentioned technique. In an event of sealer extrusion during the treatment procedure, the rate of sealer extrusion and pain was assessed separately.

\section{Post-treatment pain reduction assessment}

All the patients were handed over a pain diary form with visual analogue scale (VAS) consisting of a 100 $\mathrm{mm}$ line divided into 10 equal parts from 0 indicating no pain to 100 indicating extremely severe pain (19). This provided a range of score from 0-100, score 1-29 were graded as mild pain, 30-69 were regarded as moderate pain and 70-100 were regarded as severe pain. The patients were asked to record at $24 \mathrm{~h}, 48 \mathrm{~h}, 72 \mathrm{~h}$, and $7 \mathrm{~d}$ after treatment followed by which the patients were recalled to give the diary 
to the investigators. In case of consumption of analgesics, the type and quantity after treatment was also recorded.

\section{STATISTICAL ANALYSIS}

Data was entered in Microsoft excel spreadsheet and analyzed using SPSS software (ver. 22, IBM Corporation, Armonk. USA). The normality tests Kolmogorov-Smirnov and Shapiro-Wilks test results reveal that all variables did not follow normal distribution. Therefore, a non-parametric test was applied to analyze the data. Chi Square test was used to assess the difference in the extrusion rates among the groups. Mann Whitney U Test was used to assess the differences in the mean pain scores at different time intervals based on extrusion. Kruskal Wallis test was used to assess the differences in mean periapical lesion area and pain score between the various groups. Friedman's Two-way Analysis of Variance was used to assess the difference between the mean area of periapical lesions measured within each group at different time intervals. For the test, a $p$-value of less than 0.05 is to be considered a significance level.

\section{Results}

600 patients were checked for eligibility over a period of six months, out of which 520 did not meet the inclusion criteria and 17 were excluded because of exclusion criteria; 1 patient was pregnant, 5 had history of diabetes mellitus and 11 patients refused to participate. A total of 63 patients were available for further analysis (Fig. 1). 6 participants had not reported for follow-up appointments and hence were excluded. In conclusion data from a total of 57 patients was collected and subjected to statistical analysis (Fig. 1). There was a further loss of follow-up for 3 patients making it 9 losses of follow-up in total $(14.4 \%)$. The attrition in different groups at various time intervals is shown in Table I. Table II shows descriptive statistics, stating no statistically significant difference between the three groups based on age and gender $(p>0.05)$. There was no difference in the mean area of the periapical lesion at baseline among various groups $(p>0.05)$, this confirms comparability of the groups at baseline.

Table l: Attrition/dropout rates in assessed participants was evaluated at baseline (after completion of endodontic therapy), 1 month, 3 months and 6 months.

\begin{tabular}{|llllll|}
\hline Time interval & Tubli-Seal & AH Plus & BioRoot RCS & Total $(\mathbf{N})$ & Attrition \\
\hline Baseline & 21 & 21 & 21 & $63(100 \%)$ & 0 \\
\hline 1 week & 19 & 19 & 19 & $57(90.5 \%)$ & $6(9.5 \%)$ \\
\hline 1 month & 18 & 18 & 18 & $54(85.7 \%)$ & $9(14.3 \%)$ \\
\hline 3 months & 18 & 18 & 18 & $54(85.7 \%)$ & $9(14.3 \%)$ \\
\hline 6 months & 18 & 18 & 18 & $54(85.7 \%)$ & $9(14.3 \%)$ \\
\hline
\end{tabular}


Table Il: Characteristics of the included participants based on age and gender. No significant difference $(p>0.05)$ was seen based on gender and age for the assessed groups

\begin{tabular}{|c|c|c|c|c|c|}
\hline \multicolumn{4}{|c|}{ Treatment groups } & \multirow[t]{2}{*}{ Chi Square } & \multirow[t]{2}{*}{$\mathrm{p}$ value } \\
\hline Characteristic & Tubli-Seal & AH Plus & BioRoot RCS & & \\
\hline Age, in years (Mean) & 41.57 & 41.68 & 43.63 & 0.191 & 0.909 \\
\hline Gender, n (\%) & $12(63.2)$ & $15(78.9)$ & $11(57.9)$ & 2.053 & 0.358 \\
\hline \multicolumn{6}{|l|}{ Male } \\
\hline Female & $7(36.8)$ & $4(21.1)$ & $8(42.1)$ & & \\
\hline
\end{tabular}

The mean area of the periapical lesions in the 3 groups from baseline to the last recall is shown in Table III. The mean difference in the area of periapical lesion from baseline to 1 month for group 1,2 and 3 were $0.833,1.08$ and 1.41 respectively $(p>0.05)$. The mean difference in the area of the periapical lesion from baseline to 3 months for group 1, 2 and 3 were 4.05, 3.86 and 6.27 respectively $(p<0.05)$ (Table IV). The mean difference in the area of the periapical lesion from baseline to 6 months for group 1, 2 and 3 were $10.22,9.80$ and 13.41 respectively $(p<0.05)$ (Table IV). This shows a significant difference in the mean area of periapical lesion at 3 months and 6 months in all the three groups.

TABLE III: Mean size of the periapical lesion (in $\mathrm{mm}$ sq.) was assessed for different study groups at various time intervals (Baseline, 1 month, 3 month and 6 month). Statistical analysis using Friedman's two-way analysis of variance showed no significant difference ( $p$ value $<0.05$ ) 


\begin{tabular}{|c|c|c|c|c|c|c|}
\hline Group & Time Interval & $\mathbf{N}$ & Mean & SD ( $( \pm)$ & Chi Square & $\mathrm{p}$-value \\
\hline \multirow[t]{4}{*}{ Tubli-Seal } & Baseline & 18 & 15.25 & 13.52 & \multirow[t]{4}{*}{52.186} & \multirow[t]{4}{*}{0.001} \\
\hline & 1 month & 18 & 13.72 & 13.67 & & \\
\hline & 3 months & 18 & 10.44 & 11.04 & & \\
\hline & 6 months & 18 & 4.33 & 6.47 & & \\
\hline \multirow[t]{4}{*}{ AH Plus } & Baseline & 18 & 14.47 & 11.66 & \multirow[t]{4}{*}{52.886} & \multirow[t]{4}{*}{0.001} \\
\hline & 1 month & 18 & 11.50 & 8.87 & & \\
\hline & 3 months & 18 & 8.72 & 9.05 & & \\
\hline & 6 months & 18 & 2.77 & 5.13 & & \\
\hline \multirow[t]{4}{*}{ BioRoot RCS } & Baseline & 18 & 15.35 & 12.86 & \multirow[t]{4}{*}{52.892} & \multirow[t]{4}{*}{0.001} \\
\hline & 1 month & 18 & 13.55 & 13.26 & & \\
\hline & 3 months & 18 & 9.69 & 11.70 & & \\
\hline & 6 months & 18 & 2.55 & 4.51 & & \\
\hline
\end{tabular}

TABLE IV: The mean difference in the size of the periapical lesion at 2 time intervals for different study groups Friedman's two-way analysis of variance was done for statistical analysis and a $p$ value $<0.05$ was considered statistically significant

\begin{tabular}{|lllll|}
\hline Time interval & Healing & Mean Difference & Chi Square & P value \\
\hline Tubli-Seal & Baseline VS 1 Month & 0.833 & 0.667 & 0.137 \\
\cline { 2 - 5 } & Baseline VS 3 months & 4.05 & 1.833 & $\mathbf{0 . 0 0 0}$ \\
\cline { 2 - 5 } & Baseline VS 6 months & 10.22 & 2.778 & $\mathbf{0 . 0 0 0}$ \\
\hline \multirow{2}{*}{ AH Plus } & Baseline VS 1 Month & 1.08 & 0.722 & 0.09 \\
\cline { 2 - 5 } & Baseline VS 3 months & 3.86 & 1.861 & $\mathbf{0 . 0 0 0}$ \\
\cline { 2 - 5 } BioRoot RCS & Baseline VS 6 months & 9.80 & 2.861 & $\mathbf{0 . 0 0 0}$ \\
\cline { 2 - 5 } & Baseline VS 1 Month & 1.41 & 0.861 & 0.029 \\
\cline { 2 - 5 } & Baseline VS 3 months & 6.27 & 1.889 & $\mathbf{0 . 0 0 0}$ \\
\cline { 2 - 5 } & Baseline VS 6 months & 13.41 & 2.917 & $\mathbf{0 . 0 0 0}$ \\
\cline { 2 - 5 }
\end{tabular}

In Tubli-Seal treated group, at $24 \mathrm{~h}, 5.3 \%$ of patients showed no pain, $68.4 \%$ showed mild pain and $26.3 \%$ showed moderate pain; at $48 \mathrm{~h}, 84.2 \%$ of patients showed no pain, $10.5 \%$ showed mild pain and $5.3 \%$ showed moderate pain; At 72 h, $84.2 \%$ of patients showed no pain, $10.5 \%$ showed mild pain and $5.3 \%$ 
showed moderate pain; At $7 \mathrm{~d}$, none of the patients reported with pain. In AH Plus treated group, at $24 \mathrm{~h}$ $31.8 \%$ of patients showed no pain, $50 \%$ showed mild pain and $18.2 \%$ showed moderate pain; at $48 \mathrm{~h}$, $81.8 \%$ of patients showed no pain and $18.2 \%$ showed mild pain; at $72 \mathrm{~h}$ and $7 \mathrm{~d}$, none of the patients reported with pain. In BioRoot RCS, at $24 \mathrm{~h}, 68.4 \%$ of patients showed no pain, $15.8 \%$ showed mild pain while $15.8 \%$ showed moderate pain; at $48 \mathrm{~h}, 85 \%$ of patients showed no pain and $15 \%$ showed mild pain; at $72 \mathrm{~h}$ and at $7 \mathrm{~d}$, none of the patients had pain.

The mean pain scores at $24 \mathrm{~h}$ for Tubli-Seal, AH Plus and BioRoot RCS were 17.94 $\pm 11.35,11.57 \pm 11.18$ and $4.73 \pm 7.72$; At $48 \mathrm{~h}$ were $5.26 \pm 9.04,1.57 \pm 3.74$ and $1.57 \pm 3.74$ respectively (Fig. 2). The mean pain score at $72 \mathrm{~h}$ for Tubli-Seal was $2.63 \pm 7.33$ whereas none of the patients had pain in AH Plus and BioRoot RCS treated groups (Fig. 2). None of the patients had pain after 1-week postoperative. There was no difference $(p>0.05)$ in the mean pain score between the groups at any of the time intervals except for group 1 and group 3 at $24 \mathrm{~h}$ time interval (Table V). The extrusion rate for all the three sealers was $21.1 \%$ (Table VI). There was no significant difference in the mean pain score on the basis of presence or absence of sealer extrusion except for Group III at $48 \mathrm{~h}$ time interval (Table VII).

TABLE V: Pair wise comparison between the groups for Mean pain score at different time intervals (Group 1 - Tubli-Seal, Group 2 - AH Plus, Group 3 - BioRoot RCS). Kruskal Wallis test was done to for statistical analysis and $p$-value $<0.05$ was considered statistically significant

\begin{tabular}{|lllll|}
\hline Time interval & Groups & Mean Difference & Chi Square & p-value \\
\hline \multirow{3}{*}{ 24 hours } & Group 1 VS Group 2 & 6.36 & 9.079 & 0.235 \\
\cline { 2 - 6 } & Group 1 VS Group 3 & 13.21 & 19.342 & 0.001 \\
\cline { 2 - 5 } 48 hours & Group 2 VS Group 3 & 6.84 & 10.263 & 0.140 \\
& Group 1 VS Group 2 & 3.68 & 1.682 & 0.195 \\
\cline { 2 - 5 } & Group 1 VS Group 3 & 3.68 & 1.682 & 0.195 \\
\hline 72 hours & Group 2 VS Group 3 & 0.00 & 0.000 & 1.000 \\
\cline { 2 - 5 } & Group 1 VS Group 2 & 2.63 & 4.500 & 0.092 \\
\hline 1 week & Group 1 VS Group 3 & 2.63 & 4.500 & 0.092 \\
& Group 2 VS Group 3 & 0.00 & 0.000 & 1.000 \\
\cline { 2 - 5 } & Group 1 VS Group 3 & 0.00 & 0.000 & 1.000 \\
\cline { 2 - 5 } & Group 2 VS Group 3 & 0.00 & 0.000 & 1.000 \\
\cline { 2 - 5 } & & & 0.000 & 1.000 \\
\hline
\end{tabular}

TABLE Vl: Extrusion rates between the groups, Chi-square test was to evaluate the difference and found to be statistically insignificant $(p>0.05)$. 


\begin{tabular}{|lllllll|}
\hline Extrusion N (\%) & & $\begin{array}{l}\text { Tubli- } \\
\text { Seal }\end{array}$ & $\begin{array}{l}\text { AH } \\
\text { Plus }\end{array}$ & $\begin{array}{l}\text { BioRoot } \\
\text { RCS }\end{array}$ & $\begin{array}{l}\text { Chi square } \\
\text { value }\end{array}$ & $\begin{array}{l}\text { p- } \\
\text { value }\end{array}$ \\
\hline $\begin{array}{l}\text { Extrusion for different } \\
\text { sealers }\end{array}$ & absent & $\begin{array}{l}15 \\
(78.9)\end{array}$ & $\begin{array}{l}15 \\
(78.9)\end{array}$ & $15(78.9)$ & $0.000^{*}$ & 1.000 \\
& present & $4(21.1)$ & $\begin{array}{l}4 \\
(21.1)\end{array}$ & $4(21.1)$ & & \\
\cline { 2 - 4 } & & & & & \\
\hline
\end{tabular}

Table VII: Comparison of mean pain score based on the presence of absence of extrusion within different groups. Statistical analysis using Mann Whitney test showed significance $(p<0.05)$ only at $48 \mathrm{~h}$ for TubliSeal group.

\begin{tabular}{|c|c|c|c|c|c|c|}
\hline \multirow[t]{2}{*}{ Groups } & \multirow{2}{*}{$\begin{array}{l}\text { Time } \\
\text { intervals }\end{array}$} & \multicolumn{2}{|c|}{ Mean pain score } & \multirow{2}{*}{$\begin{array}{l}\text { Mean } \\
\text { Difference }\end{array}$} & \multirow{2}{*}{$\begin{array}{l}\text { Z } \\
\text { Value }\end{array}$} & \multirow{2}{*}{$\begin{array}{l}\mathrm{p} \text { - } \\
\text { value }\end{array}$} \\
\hline & & Present & Absent & & & \\
\hline \multirow[t]{4}{*}{$\begin{array}{l}\text { BioRoot } \\
\text { RCS }\end{array}$} & $24 \mathrm{hrs}$ & $\begin{array}{l}22.50 \pm \\
12.58\end{array}$ & $\begin{array}{l}16.73 \pm \\
11.15\end{array}$ & 5.76 & -0.903 & 0.367 \\
\hline & $48 \mathrm{hrs}$ & $10.00 \pm 8.16$ & $4.00 \pm 9.10$ & 6.00 & -1.763 & 0.078 \\
\hline & $72 \mathrm{hrs}$ & $2.50 \pm 5.00$ & $2.66 \pm 7.98$ & -0.16 & -0.473 & 0.636 \\
\hline & 1 week & $0.00 \pm 0.00$ & $0.00 \pm 0.00$ & 0.00 & 0.00 & 1.000 \\
\hline \multirow[t]{4}{*}{ AH Plus } & $24 \mathrm{hrs}$ & $17.50 \pm 5.00$ & $\begin{array}{l}10.00 \pm \\
11.95\end{array}$ & 7.50 & -1.407 & 0.160 \\
\hline & $48 \mathrm{hrs}$ & $2.50 \pm 5.00$ & $1.33 \pm 3.51$ & 1.16 & -0.553 & 0.580 \\
\hline & $72 \mathrm{hrs}$ & $0.00 \pm 0.00$ & $0.00 \pm 0.00$ & 0.00 & 0.000 & 1.000 \\
\hline & 1 week & $0.00 \pm 0.00$ & $0.00 \pm 0.00$ & 0.00 & 0.000 & 1.000 \\
\hline \multirow[t]{4}{*}{ Tubli-Seal } & $24 \mathrm{hrs}$ & $12.50 \pm 9.57$ & $2.66 \pm 5.93$ & 9.83 & -2.193 & 0.028 \\
\hline & $48 \mathrm{hrs}$ & $5.00 \pm 5.77$ & $0.66 \pm 2.58$ & 4.33 & -2.055 & 0.040 \\
\hline & $72 \mathrm{hrs}$ & $0.00 \pm 0.00$ & $0.00 \pm 0.00$ & 0.00 & 0.000 & 1.000 \\
\hline & 1 week & $0.00 \pm 0.00$ & $0.00 \pm 0.00$ & 0.00 & 0.000 & 1.000 \\
\hline
\end{tabular}

\section{Discussion}

Bone homeostasis is disturbed in an event of apical periodontitis where an increased rate of bone resorption is witnessed. During this scenario, osteoclasts, osteoblasts, osteocytes and cementoblasts are seen to be the key cells during the process of bone formation and resorption (13). The null hypothesis considered for the present study was rejected since there was significant difference in the rate of periapical healing and postoperative pain levels on using different base endodontic sealers. Periodontal ligament fibroblasts synthesize and organize collagen fibers, connecting bone to the cementum, thereby 
repairing and regenerating the periodontal structures and aiding in periapical healing (14). On the other hand, postoperative pain after root canal treatment is considered to be a localized inflammatory reaction of the periapical tissues and is considered to be directly linked to periapical healing (15). Local inflammation due to extruded sealer in the periapical region causes postoperative pain and influences the healing process with the magnitude of inflammatory reaction seen to be influenced based on the composition of sealers (11). Such inflammatory reactions are due to the release of biochemical mediators such as reactive oxygen species (ROS), which has been proved clinically to be associated with inflammatory pain (16)(17). It is noted that on direct contact with pulpal tissues the production of reactive oxygen species is increased to seven multiples when in contact with endodontic sealers (18). It is imperative that endodontic sealers used during the root canal procedure come in direct contact with periapical tissues through the apical foramen and lateral canals, thus having a potential to affect periapical healing and post-operative pain by increasing these biomedical mediator levels.

Zinc oxide eugenol based sealer such as Tubli-Seal are reported to possess increased cytotoxic and tissue-irritating potencies in in vitro cell culture studies and is shown to possess high cytotoxic potency and one of the oldest sealers used in endodontic practice (19). With the introduction of bioceramic based materials in endodontics significant strides have been made mainly as repair cements and root canal sealers (20)(13). In this study, BioRoot RCS was chosen as the experimental group since there is an unavailability of data through clinical trials suggesting its superiority in promoting periapical healing or reducing post-operative pain over other endodontic sealers. BioRoot RCS is classified as a bioactive mineral root canal sealer based on innovative mineral micro-aggregate chemistry named "active biosilicate technology" (21) and is considered to be one of the most biocompatible sealers possessing osteoinductive properties in comparison to other bioceramic based sealers $(22,23)$. Previous reports have suggested that BioRoot RCS possesses high bioactivity on human periodontal ligament cells allowing better bone modulation mechanisms to occur (24).

Endodontic therapy is known to be a complex process comprising a multitude of factors such as shaping, cleaning and obturation and thus it is very difficult to attribute the incidence of postoperative pain to any specific criteria in clinical research (25), thus there is a significant need to standardize the treatment protocol to reduce any other variable outcome. The different variables used in the present study were designed in such a manner so as to reduce as much as possible to reduce potential factors which may cause postoperative pain. Presence of preoperative pain and its intensity has been proven to have influence on postoperative pain reduction levels (26). Hence it was necessitated to conduct a multi visit procedure in spite of recent evidence suggesting tooth diagnosed with apical periodontitis treatment can be completed in a single visit procedure (27). The present study couldn't take this into consideration since preoperative pain levels could have influenced the postoperative pain levels and the use of an intracanal dressing can reduce these pain score levels thus justifying the pain incidence levels by usage of endodontic sealers itself. Therefore, only when the patients who clinically exhibited no symptoms of pain or infection, obturation was carried out. The present study included only single rooted teeth for higher degree of standardization and outcome variables can be directly correlated to the treatment outcome. It is well known that systemic diseases such as diabetes mellitus, hypertension and hormonal replacement 
affect the rate of periapical healing and thus patients with such diseases were excluded from the sample. Variability among the groups, at baseline in a randomized controlled clinical trial, might affect the value of its results. No statistically significant difference was found on the basis of age, sex, tooth type and area of the periapical lesion at baseline among the three groups indicating that they were equally distributed and comparable.

The prevalence of postoperative pain recorded in this study goes in agreement with the reports estimated by Pak and White et al.(28) who showed that prevalence of pain after endodontic is seen to be highest at 24-48 $\mathrm{h}$ and reduced at only $7 \mathrm{~d}$ interval. The overall post-operative pain prevalence at $24 \mathrm{~h}$ was $63 \%$ followed by $21 \%$ at $48 \mathrm{~h}$ followed by $5 \%$ at $72 \mathrm{~h}$ and $0 \%$ at $7 \mathrm{~d}$. Su et al.(29) had reported that postoperative pain scores post obturation were seen to be highest at $24 \mathrm{~h}$ to $48 \mathrm{~h}$ and gradually declined at $72 \mathrm{~h}$, and $7 \mathrm{~d}$ for all the assessed groups and was the factor to consider these time frame assessments for the present study. In the present study the mean postoperative pain score was seen highest for TubliSeal group, followed by AH Plus and BioRoot RCS at 24 to $48 \mathrm{~h}$ and gradually declined at $7 \mathrm{~d}$. These results could be explained by the direct cytotoxic effects of Tubli-Seal in both set and mixed state due the presence of eugenol which plays a primary role in the setting reaction (30). Jung et al.(23) had shown in their in vitro assessment that BioRoot RCS was seen to have least cytotoxic effect in both in premixed and set state whereas AH plus was reported to be only cytotoxic in its premixed state. Based on these reports, it can be concluded that their contact with periapical tissue will produce different inflammatory responses causing postoperative pain by the body's innate response to increase the production of reactive oxygen species (31) based on the leaching of different components of the sealer during the setting reaction (32).

On the account of sealer extrusion rate, it was seen to be similar $(21.1 \%)$ for all the three study groups in this present study. Though sealer extrusion during the treatment procedure is an unprecedented outcome, its influence on post-operative pain showed no significant influence based on reports by previously published studies (33). In the present study, BioRoot RCS at $48 \mathrm{~h}$ time interval showed a significant difference in mean pain score $(p<0.05)$ in comparison to other test sealers. This difference in results go in correlation with the results obtained in the in vitro cytotoxic assessment by Poggio et al.(34) in which BioRoot RCS Sealer extract showed no cytotoxicity at $24 \mathrm{~h}$, whereas mild cytotoxicity at $48 \mathrm{~h}$ and $72 \mathrm{~h}$ in comparison to epoxy-based sealers.

In the present study, the mean difference in the area of the periapical lesion for Tubli-Seal, AH Plus and Tubli-Seal were 4.05, 3.86 and 6.27 respectively at 3 months and $10.22,9.80$ and 13.41 respectively at 6 months $(p<0.05)$, suggesting better periapical healing with BioRoot RCS compared to AH Plus and TubliSeal. The results of this study can be supported by the fact that BioRoot RCS demonstrated the ability to release calcium ions (721 ppm at $3 \mathrm{~h}$ ); $\mathrm{B}$ type carbonated apatite deposits were found on aged BioRoot RCS (biointeractive-related CaP-forming ability) compared to MTA Fillapex, pulp canal sealer and AH Plus sealer (35). Release of free calcium ions produces a more pronounced differentiation of macrophages and giant cells (24), leading to better reduction of microbial infection in the periapical region, subsequently promoting healing. BioRoot RCS on the other hand has shown less toxic effect on 
periodontal ligament cells than pulp canal sealer (zinc oxide eugenol-based sealer) as it induced higher secretion of angiogenic and osteogenic growth factors; BMP-2, VEGF, and FGF-2 (24). A recent study reported that proinflammatory cytokines such as IL- 6 has been reduced and TGF-ß1 production has been increased allowing periodontal regeneration to take place (36). These mechanisms could explain the results achieved in the present study.

The periapical index (PAl) scoring was used in the present study since it gives semi-quantitative results that do not allow powerful comparison among groups (37). Thus, in this study comparison was done on the basis of the area of periapical lesion rather than the PAl scores. Measurement of area using a grid is more objective, enabling better comparability between baseline and follow-ups and reduces the chances of inter-examiner bias. The use of cone beam computed tomography is shown to accurately depict changes in cancellous bone, but an in vivo report has been established showing its sensitivity rate to be higher on detection of only healthy tooth but in conditions of a diseased state such as apical periodontitis the detection rate was seen to be similar to periapical radiography based on histological findings (38). Recent times have shown evolution of different CBCT systems exhibiting increased reduction in effective dosage rates in spite of this the dosage rates were seen to be 45 to 90 times higher in comparison to digital radiographic imaging and was not clinically applicable for the present study since the patients would have to go through multiple exposures for further evaluation (39).

Bone deposition can be considered as a clinical sign radiographically in the case of healing apical periodontitis post endodontic treatment (40). The radiographic success can be quantified by the continuous reduction in the size of a lesion over a period of time indicating success of treatment (41). Predicting the prognosis of a tooth at the earliest, after completion of endodontic treatment is a topic of interest from a clinical point of view. The evaluation of radiographic signs and clinical risk factors or a combination of both, could potentially hold importance in future clinical research. In the present study, the mean difference for the area of periapical lesion at baseline and 1 month was not statistically significant for any group, whereas the mean difference for the area of periapical lesion at baseline and 3 months; baseline and 6 months was statistically significant ( $p \otimes 0.05)$. A significant healing of periapical lesions in all the three groups at 3 months, suggests that the initial signs of the process of healing can be seen at 3 months interval. These results go in correlation with previously reported clinical study by Huumonen et al. reporting that three month control was adequate in establishing significant healing in cases of apical periodontitis when zinc oxide eugenol and silicone based sealers were used (42).

Improper disinfection of root canal, persisting bacteria in exposed dentinal tubules, lacunae of cellular cementum, apical foramen and apical extrusion of debris has shown to influence the rate of periapical healing and post-operative pain (43). A $100 \mathrm{~mm}$ VAS scale used in the present study is considered to be an effective method for the measurement of postoperative pain in clinical research (44). Although its use has been widely reported it is considered to have certain limitations such as its subjectivity depending on individual sensitivity to pain perception. One of the methods to overcome this limitation is by a split mouth design of clinical trials since the tooth of treatment is in the same participants and potentially negates these types of errors. Periapical radiographs provide a 2-D image of a 3-D bony defect. Cone 
beam computed tomography (CBCT) scan of periapical healing post endodontic treatment gave similar results to that obtained by histological microscopic analysis, whereas radiographic evaluation understated the size of the periapical lesion (45). Cone Beam Computed Tomography (CBCT) can be used for more specific evaluation of periapical healing, limiting the field of view only to the region of interest and keeping the radiation dose at the lowest.

\section{Conclusions}

Within the limitations of this study, the postoperative pain after root canal treatment using BioRoot RCS as endodontic sealer was less compared to AH Plus and Tubli-Seal. There was no significant difference in post-operative pain based on the extrusion of sealer except for BioRoot RCS at $48 \mathrm{~h}$. BioRoot RCS showed better periapical healing compared to AH Plus and Tubli-Seal at 3 months and 6 months. A period of 3 months was adequate to establish significant periapical healing in all the three groups. Further studies are required with other different base endodontic sealers to further justify the results obtained in this study.

\section{Abbreviations}

NaOCl: Sodium hypochlorite

EDTA: Ethylenediaminetetraacetic acid

PAl: Periapical index

No.: Number

s: Seconds

h: Hour

ppm: parts per million

СBCT: Cone-beam computed tomography

VAS: Visual analogue scale

mm: Millimeter

\section{Declarations}

\section{Ethics approval and consent to participate}

Ethical approval was obtained from the Institutional Review Board (SRB/SDMDS11/170DS/09). All the patients consented to an informed consent form discussing the details of the present study as well as 
benefits and risks of the study.

\section{Ethics approval was done from}

Saveetha Dental College, Saveetha University, Chennai, Tamil Nadu, India

\section{Consent for publication}

All the authors have significantly contributed to the various stages of the present study and all in agreement with publication of the manuscript.

\section{Availability of data and material}

Data can be shared upon contacting the corresponding author on reasonable request

\section{Competing interests}

None

\section{Funding}

None

\section{Author's contributions}

Conceptualization - A.K, A.P, Study Design: A.K, J.J, V.T, Data collection - A.K, J.J, V.T, Supervision - A.P, Materials - A.K, Literature Search - J.J, V.T, A.K, Original Draft: A.K, Critical Review - J.J, V.T

\section{Acknowledgments}

The authors would sincerely thank Dr. Zoha Abdullah for significant help for conducting the statistical analysis for the present study.

\section{References}

1. Siqueira JF Jr, Rôças IN. Microbiology and treatment of acute apical abscesses. Clin Microbiol Rev. 2013 Apr;26(2):255-73.

2. Ng YL, Mann V, Rahbaran S, Lewsey J. Outcome of primary root canal treatment: systematic review of the literature-Part 2. Influence of clinical factors. International Endodontic Journal. 2008; Available from: https://onlinelibrary.wiley.com/doi/abs/10.1111/j.1365-2591.2007.01323.x

3. Zehnder M. Root Canal Irrigants. Vol. 32, Journal of Endodontics. 2006. p. 389-98. Available from: http://dx.doi.org/10.1016/j.joen.2005.09.014

4. Peters OA, Peters Cl. Cleaning and Shaping of the Root Canal System. Cohen's Pathways of the Pulp. 2011. p. 283-348. Available from: http://dx.doi.org/10.1016/b978-0-323-06489-7.00009-6 
5. Lee JK, Kwak SW, Ha J-H, Lee W, Kim H-C. Physicochemical Properties of Epoxy Resin-Based and Bioceramic-Based Root Canal Sealers. Bioinorg Chem Appl. 2017 Jan 22;2017:2582849.

6. Dalopoulou A, Economides N, Evangelidis V. Extrusion of Root Canal Sealer in Periapical Tissues Report of Two Cases with Different Treatment Management and Literature Review. Vol. 21, Balkan Journal of Dental Medicine. 2017. p. 12-8. Available from: http://dx.doi.org/10.1515/bjdm-20170002

7. Berbert FLCV, Leonardo MR, Silva LAB, Tanomaru Filho M, Bramante CM. Influence of root canal dressings and sealers on repair of apical periodontitis after endodontic treatment. Oral Surg Oral Med Oral Pathol Oral Radiol Endod. 2002 Feb;93(2):184-9.

8. AlRahabi MK. Predictors, prevention, and management of postoperative pain associated with nonsurgical root canal treatment: A systematic review. J Taibah Univ Med Sci. 2017 Oct;12(5):37684.

9. Sathorn C, Parashos P, Messer H. The prevalence of postoperative pain and flare-up in single- and multiple-visit endodontic treatment: a systematic review. Int Endod J. 2008 Feb;41(2):91-9.

10. Genet JM, Hart AA, Wesselink PR, Thoden van Velzen SK. Preoperative and operative factors associated with pain after the first endodontic visit. Int Endod J. 1987 Mar;20(2):53-64.

11. Zhang W, Peng B. Tissue reactions after subcutaneous and intraosseous implantation of iRoot SP, MTA and AH Plus. Dent Mater J. 2015;34(6):774-80.

12. Paulaian B, Thakur S, Emil J. Evaluation of mineral trioxide aggregate as root canal sealer: A clinical study. Vol. 16, Journal of Conservative Dentistry. 2013. p. 494. Available from: http://dx.doi.org/10.4103/0972-0707.120944

13. Loi F, Córdova LA, Pajarinen J, Lin T-H, Yao Z, Goodman SB. Inflammation, fracture and bone repair. Bone. 2016 May;86:119-30.

14. Lekic P, McCulloch CAG. Periodontal ligament cell populations: the central role of fibroblasts in creating a unique tissue. The Anatomical Record: An Official Publication of the American Association of Anatomists. 1996;245(2):327-41.

15. Omoigui S. The biochemical origin of pain: the origin of all pain is inflammation and the inflammatory response. Part 2 of 3 - inflammatory profile of pain syndromes. Med Hypotheses. 2007 Aug 28;69(6):1169-78.

16. West AP, Shadel GS, Ghosh S. Mitochondria in innate immune responses. Nat Rev Immunol. 2011 Jun;11(6):389-402.

17. Hackel D, Pflücke D, Neumann A, Viebahn J, Mousa S, Wischmeyer E, et al. The connection of monocytes and reactive oxygen species in pain. PLoS One. 2013 May 2;8(5):e63564.

18. Camargo CHR, Camargo SEA, Valera MC, Hiller K-A, Schmalz G, Schweikl H. The induction of cytotoxicity, oxidative stress, and genotoxicity by root canal sealers in mammalian cells. Oral Surg Oral Med Oral Pathol Oral Radiol Endod. 2009 Dec;108(6):952-60.

19. Geurtsen W. Biocompatibility of root canal filling materials. Aust Endod J. 2001 Apr;27(1):12-21. 
20. Atmeh AR, Chong EZ, Richard G, Festy F, Watson TF. Dentin-cement interfacial interaction: calcium silicates and polyalkenoates. J Dent Res. 2012 May;91(5):454-9.

21. Taraslia V, Anastasiadou E, Lignou C, Keratiotis G, Agrafioti A, Kontakiotis EG. Assessment of cell viability in four novel endodontic sealers. Eur J Dent. 2018 Apr;12(2):287-91.

22. Dimitrova-Nakov S, Uzunoglu E, Ardila-Osorio H, Baudry A, Richard G, Kellermann O, et al. In vitro bioactivity of Bioroot ${ }^{\mathrm{TM}}$ RCS, via A4 mouse pulpal stem cells. Dent Mater. 2015 Nov;31(11):1290-7.

23. Jung S, Sielker S, Hanisch MR, Libricht V, Schäfer E, Dammaschke T. Cytotoxic effects of four different root canal sealers on human osteoblasts. PLoS One. 2018 Mar 26;13(3):e0194467.

24. Camps J, Jeanneau C, El Ayachi I, Laurent P, About I. Bioactivity of a Calcium Silicate-based Endodontic Cement (BioRoot RCS): Interactions with Human Periodontal Ligament Cells In Vitro. J Endod. 2015 Sep 1;41(9):1469-73.

25. Ng Y-L, Mann V, Gulabivala K. Outcome of secondary root canal treatment: a systematic review of the literature. Vol. 41, International Endodontic Journal. 2008. p. 1026-46. Available from: http://dx.doi.org/10.1111/j.1365-2591.2008.01484.x

26. Alí A, Olivieri JG, Duran-Sindreu F, Abella F, Roig M, García-Font M. Influence of preoperative pain intensity on postoperative pain after root canal treatment: A prospective clinical study. J Dent. 2016 Feb;45:39-42.

27. Orstavik D. Essential Endodontology: Prevention and Treatment of Apical Periodontitis. John Wiley \& Sons; 2020. 408 p.

28. Pak JG, White SN. Pain prevalence and severity before, during, and after root canal treatment: a systematic review. J Endod. 2011 Apr;37(4):429-38.

29. Chu $\mathrm{C}-\mathrm{H}$, Wong A, Zhang $\mathrm{C}$. A systematic review of nonsurgical single-visit versus multiple-visit endodontic treatment. Clinical, Cosmetic and Investigational Dentistry. 2014. p. 45. Available from: http://dx.doi.org/10.2147/ccide.s61487

30. Szczurko G, Pawińska M, Łuczaj-Cepowicz E, Kierklo A, Marczuk-Kolada G, Hołownia A. Effect of root canal sealers on human periodontal ligament fibroblast viability: ex vivo study. Odontology. 2018 Jul;106(3):245-56.

31. Victoria-Escandell A, Ibañez-Cabellos JS, de Cutanda SB-S, Berenguer-Pascual E, Beltrán-García J, García-López E, et al. Cellular Responses in Human Dental Pulp Stem Cells Treated with Three Endodontic Materials. Stem Cells Int. 2017 May 24;2017:8920356.

32. Lodienė G, Kopperud HM, Ørstavik D, Bruzell EM. Detection of leachables and cytotoxicity after exposure to methacrylate- and epoxy-based root canal sealers in vitro. Vol. 121, European Journal of Oral Sciences. 2013. p. 488-96. Available from: http://dx.doi.org/10.1111/eos.12065

33. Chybowski EA, Glickman GN, Patel Y, Fleury A, Solomon E, He J. Clinical Outcome of Non-Surgical Root Canal Treatment Using a Single-cone Technique with Endosequence Bioceramic Sealer: A Retrospective Analysis. J Endod. 2018 Jun;44(6):941-5.

34. Poggio C, Riva P, Chiesa M, Colombo M, Pietrocola G. Comparative cytotoxicity evaluation of eight root canal sealers. J Clin Exp Dent. 2017 Apr;9(4):e574-8. 
35. Siboni F, Taddei P, Zamparini F, Prati C, Gandolfi MG. Properties of BioRoot RCS, a tricalcium silicate endodontic sealer modified with povidone and polycarboxylate. Int Endod J. 2017 Dec;50 Suppl 2:e120-36.

36. Jeanneau C, Giraud T, Laurent P, About I. BioRoot RCS Extracts Modulate the Early Mechanisms of Periodontal Inflammation and Regeneration. Vol. 45, Journal of Endodontics. 2019. p. 1016-23. Available from: http://dx.doi.org/10.1016/j.joen.2019.04.003

37. Akdeniz BG. Evaluation of periapical lesion healing by correction of gray values. J Endod. 2005 Jul;31(7):487; author reply 487.

38. de Paula-Silva FWG, Wu M-K, Leonardo MR, da Silva LAB, Wesselink PR. Accuracy of periapical radiography and cone-beam computed tomography scans in diagnosing apical periodontitis using histopathological findings as a gold standard. J Endod. 2009 Jul;35(7):1009-12.

39. Kadesjö N, Lynds R, Nilsson M, Shi X-Q. Radiation dose from X-ray examinations of impacted canines: cone beam CT vs two-dimensional imaging. Dentomaxillofac Radiol. 2018 Feb;47(3):20170305.

40. Kerosuo E, Orstavik D. Application of computerised image analysis to monitoring endodontic therapy: reproducibility and comparison with visual assessment. Dentomaxillofac Radiol. 1997 Mar;26(2):79-84.

41. Bystrom A, Happonen RP, Sjogren U, Sundqvist G. Healing of periapical lesions of pulpless teeth after endodontic treatment with controlled asepsis. Endod Dent Traumatol. 1987 Apr;3(2):58-63.

42. Huumonen S, Lenander-Lumikari M, Sigurdsson A, Ørstavik D. Healing of apical periodontitis after endodontic treatment: a comparison between a silicone-based and a zinc oxide-eugenol-based sealer. Int Endod J. 2003 Apr;36(4):296-301.

43. Yusuf $H$. The significance of the presence of foreign material periapically as a cause of failure of root treatment. Oral Surg Oral Med Oral Pathol. 1982 Nov;54(5):566-74.

44. Arias A, de la Macorra JC, Hidalgo JJ, Azabal M. Predictive models of pain following root canal treatment: a prospective clinical study. Int Endod J. 2013 Aug;46(8):784-93.

45. Paula-Silva FWG de, de Paula-Silva FWG, Júnior MS, Leonardo MR, Consolaro A, da Silva LAB. Conebeam computerized tomographic, radiographic, and histologic evaluation of periapical repair in dogs' post-endodontic treatment. Vol. 108, Oral Surgery, Oral Medicine, Oral Pathology, Oral Radiology, and Endodontology. 2009. p. 796-805. Available from: http://dx.doi.org/10.1016/j.tripleo.2009.06.016

\section{Figures}




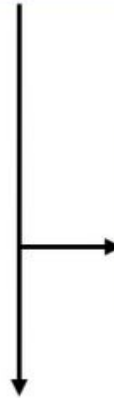

\section{Excluded $=537$}

Not meeting inclusion criteria $(n=520)$

Due to exclusion criteria $(n=17)$

\section{Randomised $(n=63)$}
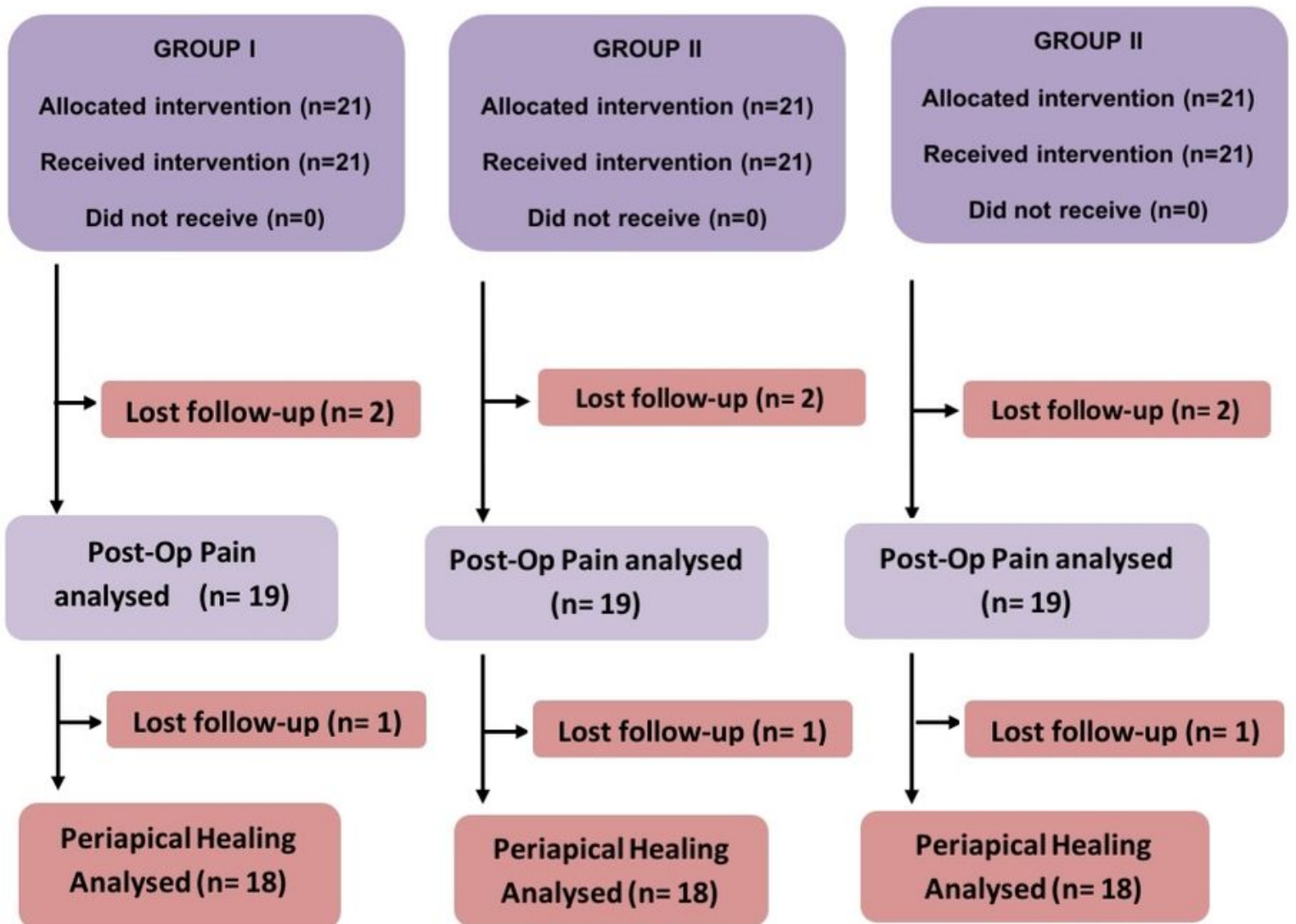

\section{Figure 1}

CONSORT flowchart showing the design of the present study with overview of the different treatment protocols, loss of patients to follow-up and periapical healing analysis 


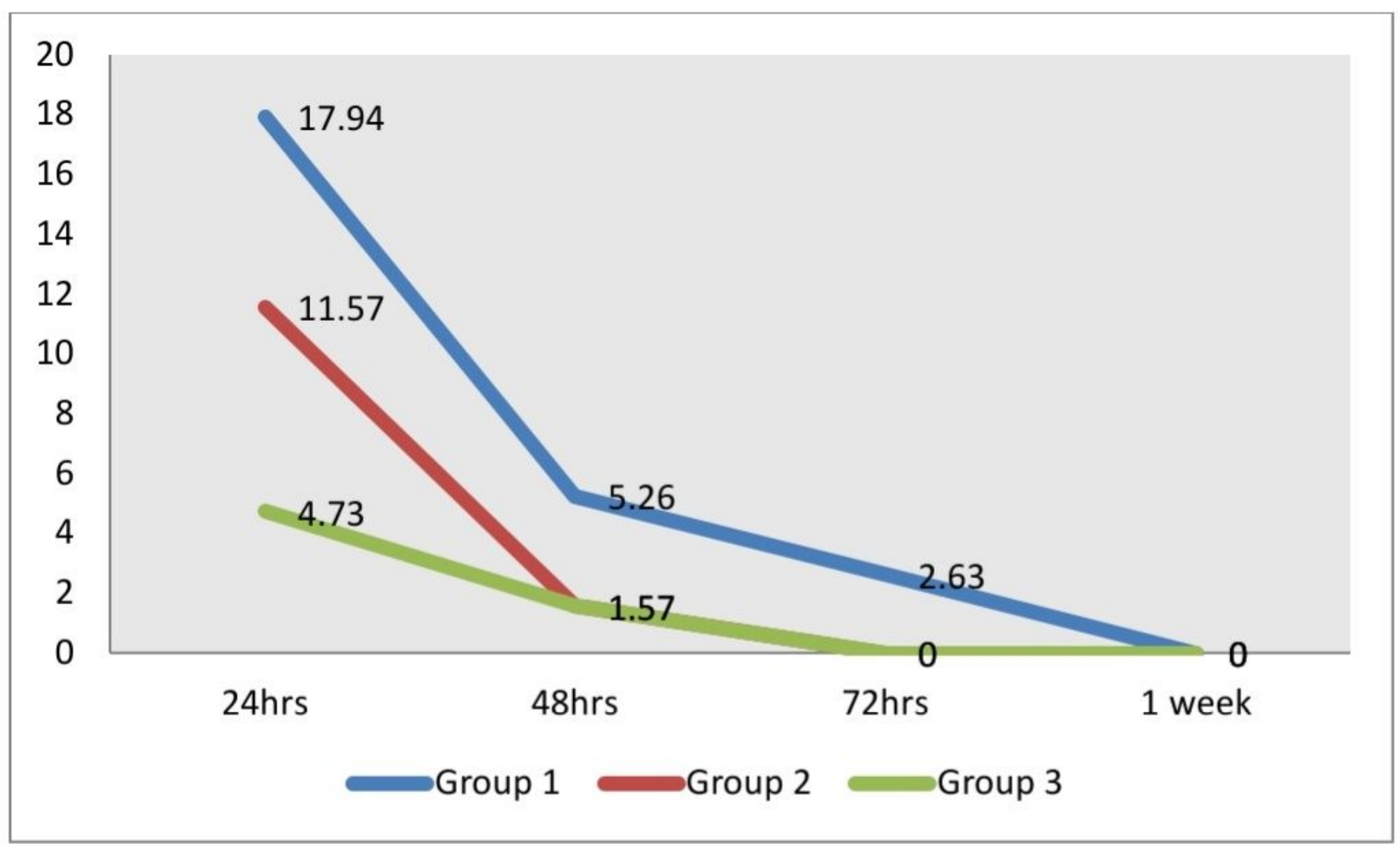

Figure 2

The mean postoperative pain scores were evaluated at different time intervals ( $24 \mathrm{~h}, 48 \mathrm{~h}, 72 \mathrm{~h}$, and $7 \mathrm{~d}$ ) using the 100mm VAS Score. (Group 1 - Tubli-Seal, Group 2 - AH Plus, Group 3 - BioRoot RCS)

\section{Supplementary Files}

This is a list of supplementary files associated with this preprint. Click to download.

- CONSORT2010Checklist.doc 